WHAREHUIA MILROY

(Transcribed by Jason King)

\title{
He Poroporoaki ki a Te Rere Amoamo (Monte) Ohia Nā Te Wharehuia Milroy
}

\begin{abstract}
I whakaeke a Te Wharehuia $i$ te ope $i$ tae atu ki runga $i$ te marae o Waikawa $i$ te taha o Te Rere Amoamo, arā, o Monte Ohia. I haria mai te tūpāpaku $i$ Ōtautahi ki te marae o tana wahine. Ka mutu ngā whaikōrero a te tangata whenua, a Te Äti Awa, $i$ roto $i$ te wharenui, ka tū atu a Awanui Black nō Ngāti Pükenga, te iwi o Te Rere Amoamo, ki te wāhi i ngā kōrero mō te manuhiri. Ka whai mai a Hohepa Williams, kātahi ka tū atu ai a Te Wharehuia me tana poroporoaki e whai ake nei.
\end{abstract}

Ngā kāwai ariki kua tae mai nei ki te whare kāhui ariki, tēnā koutou, tēnā koutou, tēnā koutou ki roto i tēnei te hāora o ō tātau pouritanga.

Tuarua, ka mihi ake, ki a tātau katoa. Tātau e kao mai nei i waho i tō tātau marae i runga i te reo karanga o te tangata nei, kotahi tonu te kaupapa i whāiti mai ai tātau ki roto i tō tātau whare, ā, koia tēnei, e takoto ake nei.

Tuatoru, ki a koutou te hunga nā koutou i taki mai tō tātau mate, mai i roto i Waitaha, à, tatū noa mai nei ki konei, tēnā koutou i roto i ngā whakaritenga o te wā. Tēnā koutou i takahi mai nei i te nuku o te whenua, kia tatū mai nei koutou ki roto ki tēnei, tō tātau marae, kia noho tahi ai tātau, kia kōrerotia e tātau, kia tangihia e tātau te āhua o te tangata e takoto ake nei.

E Whakateurunga te moenga te whāriki huaranga tonu atu ki Rūāmoa aku tīpuna e kiia mai nei, he tūtūa, he tautauhea, he moenga raukawakawa. Nukunukutia ai te tangata ki tahaki kia wātea he nohoanga mō te tangata tiketike, kumekume mātāwaka ki runga i te marae kia tangi te pīpīi runga i te rangi, ù è! auē! Tìhei mauriora!

Formerly the professor of the Department of Maori at The University of Waikato, Wharehuia Milroy is now a member of the Waitangi Tribunal and Te Panekiretanga o Te Reo, which aims to promote excellence in the Maori language. He was awarded a QSO in 2003 and an Honorary Doctorate from The University of Waikato in 2005. 
Te Rere Amoamo, kātahi anō nei ka rongo i tēnei ingoa àtaahua ōu. Kia mate rawa koe, kātahi anō ka whakaputaina te ingoa nei me te pātai i roto i a au, 'He aha koe i huna ai i tēnei ingoa?' I te mea, kai roto e takoto ana ngā whakamāramatanga e kōrerotia ake nei e ngā kaikōrero i te pō nei, ngā āhuatanga katoa o te ao i takahia ai e koe i roto i tōu wā.

Nō reira, Te Rere Amoamo, ko koe te waka whakarei kua pae ki uta, anā, kua tae mai koe i tēnei pō ki roto i te poho o te whare nei o Arapāoa. Nō reira, kōrerotia ake nei te āhua ki a koe. Te kōtuku noho awa kua rere, te hai māpuna kua riro tītapu, te whare kōrero o tō iwi o Ngāti Pūkenga - kua wahangū, kua kī kore, kua taringa turi.

Te Rere Amoamo, ānei he kōrerotia ake nei te āhua ki ngā mahi i mahia ai e koe.

Te kaupapa o tēnei mea o te mātauranga i uru koe ki ngā whare wānanga katoa. E taea te kî, ko tēnei motu, nāu i whāwhā ngā take o roto i ērā whare wānanga. Nō tēnei whare wānanga e takoto ake nei koe, ka waiho koe i ngā kōrero mā ètahi kē e kōrero. Koinei, anake te whare wānanga e whakatutukihia ai e koe i roto i te anu, i te mātaotao. Kāore i whakatutukihia e koe i roto $i$ te āhua o te kirimaha, engari nā tēnei kātahi anō ka kitea. Anei e tau ake nei, ngā hunga kua haere mai, ki te whai mai i tō reo i puhaina ai e koe, te hā whakamutunga o roto o tō tinana.

Nā reira, Te Rere Amoamo, kua riua koe i te au o oti atu, ki whakangaro, ki te kāinga i karangahia mai ai koe e ō koroua, e ō kuia, ka whakarongo ake ki te āhua o ngā whakapapa e rārangihia ana e tō kaikōrero e Te Awanui. Ka tangi ake te ngākau i roto i a au, i te mea, kai te whakarongo ake au, kia pēheatia e au? E kōrero ake ana ki te āhua o tō haerenga mai ki kōnei me ngā taukumekume i roto i ngā rā, engari, me pēnei te kōrero, hai whakautu i ètahi o ngā pātai a te rangatira o roto o Te Āti Awa nei. Kua oti te rārangi atu tō whakapapa mai i a Weka rāua ko Irakewa, ka taka mai ki a Toroa, ka moe i a Pūhounui ka puta ko Wairaka. Kātahi rāa Turi me tana iwi o roto o Taranaki, ka haere ake ki te Whare Wānanga o Mātaatua i roto o Whakatāne ko Tūpāpakurau te ingoa o te whare wānanga nei. Te haerenga ake ka haere mai te tangata nei ko Maiurenui te ingoa. 
Te Rere Amoamo, kai te whaiwhai ake nei i ēnei kōrero nā te mea he wāhanga ki te āhua e takoto nei koe i konei. He wāhanga anō ki te āhua o te whakaaro kia whakahokia koe ki roto i te rohe o Mātaatua.

Te taenga mai o te Maiurenui nei ko te tangata pai ki te kōrero i ēnei kōrero nō inanahi tonu nei i mate ai, ā, ko te Hautūtūa. Mōhio kē ake ki te rūkahau i a au. Heoi anō, e taka te wā ka whakaaro te matua o Wairaka (e noho puhi ana a Wairaka) ka whakaaro te matua o Wairaka, ā, me tohu ai e ia he tāne māna. I te mea kua tatū mai he ope o roto o Te Āti Awa ki roto o Whakatāne. Te kaupapa i haere mai ai, ko ngā mahi o èrā wā he whakawhitiwhiti i ngā kōrero o roto i ngā whare wānanga o tērā wā. Pēnei anō i te tangata e takoto ake nei. Kia haere ai ki roto i ngā whare wānanga o tēnā wāhi, o tēnā wāhi, o tēnā wāhi ki te whāngai i te mātauranga ki te hunga e taringa areare atu ana. Heoi anō, ka tae mai ki roto o Whakatāne, ā, ka noho, à, ka whakaae te wahine rā māna e tohu tana tāne (pēnei pea te āhua i a Pou nei - he àtaahua), engari ka tae mai a Maiurenui. E mōhio ana a Wairaka ko wai tana tāne e hiahia ana ia, nā reira, ka takoto mai te tangata rā ki tētahi taha o te whare, kai tētahi taha o te whare te Wairaka nei nā, kai te kopa iti. Kai korā ngā rangatira e noho ana, ā, ka noho mai te tāne, te tangata ātaahua, heoi anō, ko ngā taringa o Maiurenui kua rongo kē i te kōrero a Wairaka 'Māku e tohu taku tāne, ki te rarapi ahau i te pāpāringa o taua tangata rā, ka kite koutou i ngā haehaetanga o ōku matimati ki runga ki tana pāpāringa'.

I tāua pō, ka kite atu te tangata rā a Maiurenui, kātahi ka ngōki atu i roto i te pōuri, ka kōrero atu ki te taringa o te tangata rā, 'E hoa, tēnā koa ko au he āhua mātao nōku, ko au ki konā, ko koe ki korā'. Kāore te tangata rā e mōhio ana kai te tohua ia hai tāne mā Wairaka. Heoi anō, ao ake i te ata ka karangatia ngā iwi kia huihui ki waho, ka tū mai a Toroa ki te pānui i ana kōrero, ā, kātahi ka pānuitia, kua tohua e tana tamāhine tana tāne anei, te tohu, kua haehaetia te pāpāringa. Kātahi ka huri haere, ka kitea ko Te Ati Awa i toa. Kai reira, e mau ana te haenga a ngā ringa a ngā matimati o Wairaka.

Kaua e titiro ki te āhua o te kōrero, engari, kai te whaiwhai kē atu ki te wairua o ā tātau kōrero, e kōrero ake nei tātau i te pō nei, ki ngā whanaungatanga ki roto, ki waenganui i a tātau, 
ki ngā kaupapa i pēnei ai te āhua o tā tātau noho. Taihoa au whaiwhai ake ōku whakaaro nā te mea, he pātai āku engari waiho ērā mō muri, kia mutu taku poroporoāki ki tō tātau rangatira e takoto ake nei.

Tēnei tangata e kī ake nei ahau, i hīkoi ai i roto i ngā whare wānanga katoa. Ka hoki aku whakaaro ki te tau i tīmataria ai tētahi kaupapa e te rōpū o te Tohu Mātauranga o Aotearoa NZQA. Nā, he wāhanga Māori i roto i taua kaupapa rā, ana, ko te tangata nāna i whakatokoto taua kaupapa, anei, ko Te Rere Amoamo nei, kai konei anō a Te Rewa, kai konei a Trevor, engari koinei ngā tāngata i roto i tērā wā e kōrero ana i te kaupapa a te NZQA. Kai te hoki au ki ngā tau o te waru tekau, o tērā rautau i kōrerotia ai e Montē, i horapa ai tērā kaupapa ki te motu whānui.

Ko ngā ringaringa o te tangata nei, ko ngā kaitakitaki, ko ngā kaiārahi, i whakanohonohoia e ia he huihuinga tāngata hai mahi i aua mahi rā. Ko ia tēnei ko ngā whakaaro o te tangata nei, he tika ngā kōrero nei, kāore i pae ki a ia anake ngā kōrero, engari, i tiro whakawaho kē ki te iwi Māori me te whakaaro ake i roto i a ia, kia kitea e ia he oranga tangata. Ka whāia e ia ko taua kaupapa rā. I roto noa anō i ngā tau poto kua mahue ake nei, ka whakatūria mai anō e ia tētahi kaupapa i roto, anei, o Waitaha. Ka noho ko ia te kaumātua o roto o tērā o ngā whare wānanga, engari ka whakatūria e ia he rōpū. He mea whakakao mai e ia mō ngā tōpito ō ngā motu nei, hai kōrero i ètahi āhuatanga e taea ai te whakaaro ake, hai tautoko i ngā iwi Māori kai roto o Te Waipounamu e noho ana. Ko ia tēnei ko te āhua o te tangata kāore i mutu noa mai ki reira. Anei e kōrerohia ake nei e Ngāti Pūkenga te āhua ki tō rātau tangata. Nā reira Montē, te ingoa e matatau rā ana te nuinga, he aha he kōrero? Mēnā e taea ana e au te haere te whaiwhai i te tangata i a Aituā, ka haere au kia riro māku tonu hai kōwhaki i tana kauae. Ka pātai atu au ki a ia, he aha i pēneitia ai mātau te iwi Māori e koe? E hāua nei mātau e koe, te tāhuri kē ai ki ētahi iwi kē. Engari, ko te iwi Māori ko mātau ēnei e tākirikiritia nei e tō ringa. Koinei te aroha o ngā mea tonu o roto o te ao Māori. E mōhio ana anō tātau he tangata e taea ana te arahi i a tātau, koinei tonu ngā mea e huti ana i te ringa kaha o Aituā. Nā reira, koinei ngā tangi ake, ā, me pēnei te kōrero, tō mate he mate whakaiwikore 
tangata. Pēnei anō i a Hohepa nei, ka rongo ahau kua hinga a Montē ko tōku whakaaro ko tō matua. Ka rua, ka toru aku uiui ki te tangata, kia mātau au e tika ana tērā kōrero, kua kōrerotia mai, e, ko koe kē tēnā kua tiraha nei.

Nā reira, koinā ka kī ake au e whakaiwikore ana $\mathrm{i}$ te tangata, he whakahaehae $i$ te whakaaro, he whakamatakerekere i te hinegaro, e kore e taea e au ètahi whakaaro tūturu te whakanoho, engari e rere āmio kē ana ngā whakaaro. Koia ngā āhuatanga ka pā mai ki te motu nei i tēnei wā, koinei e kite ake nei tātau katoa, ka tere rāoa, ka tere pīpi whākao te motu ki runga i te tangata nei. Nō reira Montē, ka hoki au ki ngā kōrero i pātaihia, mā wai e whakaara te iwi ka hē? Koinei te āhua kua mahue i te Pāti Māori i ngākaunui nei koe ki te whai. I ngākaunui ai kia uru atu koe ki roto, kia pai ai tō whakapuaki ki te aroaro o te Whare Pāremata i ngā mate e whakawhiu ana ki runga i te iwi Māori. Ahakoa kai reira ō tangata, a Te Ururoa, a Hone, a Tariana, a Pita. He aha ia te hē o tētahi atu waha ki te taha i ō rātau kia tuaruatia ai te whakaaro? Koinei te āhua o tō mate, ka pai koe ki runga i te marae nei ka kite i ngā kara ki runga i ngā waka e iri ana. Ka kapakapa te ngākau. E kapakapa ana nō te mea, ahakoa kua riro koe engari ko te wairua kai te ora tonu. Nā reira, haere Ngāti Pūkenga, te taonga a tō iwi ki te motu nei, ahakoa nō te waka o Mātaatua, ka kōrero au i tērā kōrero, nā Ngāti Pūkenga te tangata nei i tono ki ngā wahi e ngoikore ana te iwi Māori. Nā reira, takahia atu te ara maioha, mai rā i ō matua i ō tîpuna ki a koe. Takahia atu ngā tapuwae ō mātua ō tīpuna, kua tūhia ō tapuwae ki ngā one o te pae ō maumahara. E kore e wareware, e kore e wareware te hunga e ora nā i tēnei wā.

Nō reira, e tō mātau hoa, e te tangata i whakatūria ai hai pou matua i roto o Waitaha, me aha rā mātau ināianei? Nō reira haere koe, haere ki ō mātua, ki ō tīpuna. Ā kāti ake, ki a koe Te Rere Amoamo, ki te whānau pani kai te mōhio koutou katoa ki te āhua o tēnei mea te mate te kākahu taratara kua ūhia ki runga i a koutou. Ehara koutou i te whānau wehewehe, he whānau piri koutou, pēnei anō i te kōrero ā te tangata, koinei te wā e kitea ai te huihuinga o Matariki e huihui nei tātau i roto i tēnei wā. Koia tēnei ko tātau kua tae mai ki te tauawhi i ngā whakaaro kai roto i tēnā, i tēnā, i tēnā, 
e aroha ana ki te tangata nei, nā reira, ko koutou, ko te whānau, whakaarohia ake tēnei kōrero: ko te ata o te piri tēnei i haere mai ai te anu mātao hai hahau i te tangata, anei e kōrerotia ake nei ngā mate tūātini o tērā whaitua, o tēnā marae, o tēnā kāinga. Nā reira, ki a koutou, kia kaha koutou i tēnei wā, Watson, Bentham, Te Kowhai, e Hone, e aroha ake ana ki a koe e noho mai nei, koutou o te whānau nei, e aroha ana nō te mea i kī ake nei au kāore kē tātau e mōhio ka tau te ringa o aituā ki hea i roto i te wā poto noa iho nō reira, kai te ngāpu tonu te whenua, kai te ngāpu tonu te whenua. Ā koinei te tohu o tēnei mea o te tangata rongonui $\mathrm{i}$ takahia ai te whenua e te tini e te mano. Koutou o te kirimate o te whare mate, tēnā koutou ngā puna roimata te hunga e whakarite ana $\mathrm{i}$ te wāhi $\mathrm{i}$ te takoto o te tangata nei, āe, awhitia, whakamahanatia kia ngaro rawa i te tirohanga kanohi.

$\mathrm{E}$ te pae, tēnā koutou e hui mai nei. Ka hoki ngā whakaaro, kia hoki ake au ki te āhua o tō kōrero o Takere rāua ko Mumuhau. Nā tētahi o ngā kaupapa i haere mai ai, tō koutou rangatira a Turi ki roto o Mātaatua he whakawhitiwhiti whakaaro. E kī ana Mātaatua, kai te mōhio ake, he kōrero anō tā Tainui, engari e kī ana a Mātaatua i poua te mauri o Mumuhau rāua ko Takeretoa ngā manu nā rātau i arahi mai i ngā waka nei.

Hei whakaoti atu i aku kōrero, he whakaaro tēnei, Bentham, Watson, ki a tātau katoa. Kāore i te hoki ki te whakawā i te āhua o ngā kōrero i whakatakotoria ai e koutou mō te tangata nei, engari e hoki kē ana ki te āhua o ngā tikanga kai te tuitui i a tātau katoa. Kua tae pea tēnei ki te wā hai āta noho mā tātau, mā ngā iwi hai whakawhitiwhiti whakaaro ki runga i ō tātau mate. I roto i ngā rā poto ka taha atu nei e kōrerotia ana te āhua ki te iwi o te Ūpokorehe me ōna mate i raru ai. Ehara i te mea i whāiti kē ana ki a rātau, engari kua tīmata tēnei āhua me te pātai ko tēhea te huarahi hai whainga mā tātau. Ko tēhea te huarahi e tika ana hai whakatakoto mā tātau, inā hoki, ki tōku mōhio mēnā i kōtia te pito ki wāhi kē ka hoki ki reira. Tuarua, kāore i te whānau te tikanga, engari kai te hapū kē. Tuatoru, kua tae mai tātau ki te ao hurihuri nei, ko te kimi kē i tētahi huarahi, e kore ai e tino tīwekaweka te āhua o ngā whakaaro, engari ka kitea tētahi huarahi e rarata ana ki te katoa. 
Heoi anō e koa ana te ngākau, i tū ake a Te Awanui ki te whakapuaki i ōnā whakaaro. Koinei ō mātau tūākana, kāore hoki i puta i a ia tērā wāhanga o tō mātau whakapapa i a rāua tahi ko Joe. He wāhi iti noa iho hai whakaoti ake i aku kōrero ka moe a Iraweka i a Weka ka puta ko te Toroa nei, ka moe i a Puhounui kia puta ko Wairaka, ka moe i a Maiurenui ka puta ko Tamatea-ki-te-huatahi. Ka moe i a Paewhiti, e whā ngā tamariki ko Ueimua kai Whakatāne e noho ana, ko Tāne-moeahi ka moe i tana wahine i a Hiwarau. Anei kia puta ko Pūkenga nāna ko Whetū, anei, te kāinga o te tangata nei. Heoi anō, kai te kite mai koutou hai tūākana ēnei ki a mātau. Nā Tane-moe-ahi nō muri mai ko Tūhoe Pōtiki nō muri i a Tūhoe Pōtiki, ko tō rātau tuahine ko Uenukurauiri, nā reira koinei te noho o te waka nei $\mathrm{i}$ tēnei wā $\mathrm{i}$ runga $\mathrm{i}$ te karangatanga o te tūākana, o te tāina (mō tēnei wā noa iho).

Nō reira, kāti ake ēnei kōrero, āpiti hono tātai hono, rātau te hunga mate kua herea ki te taura hererua - rātau ki a rātau. Tātau te hunga ora e noho ake nei i roto i ngā pīkautanga o te wā tātau ki a tātau. Kua tae mai i roto i te hunga ora, Bentham, a Te Panekiretanga. Kua tae mai i roto $i$ te hunga ora te kanohi o Te Kōhanga reo ngā kaupapa i whāia ai e tō koutou matua i tautokongia ai e tō koutou matua.

Nā reira, kāti ake i tēnei wā tēnā koutou, tēnā koutou, tēnā tātau katoa. 